\title{
A piezoresistive flexible sensor to detect soft actuator deformation
}

\author{
Martina Maselli, Debora Zrinscak, Vanni Magliola, and Matteo Cianchetti, Member, IEEE
}

\begin{abstract}
A textile-based strain sensor for measuring the length of a McKibben pneumatic actuator has been developed. McKibben actuators are flexible, lightweight, and widely used in all those applications where compliance and safety are required, e.g. soft robotics and power assisting device. The actuator length needs to be measured to control the device accurately. However, properties such as flexibility and lightness might be lost if rigid sensors such as potentiometers or linear encoders are directly attached to the actuators. For this reason, flexible and stretchable sensors are necessary. In this study, a flexible sensor using conductive textile is proposed to actively measure the length of manufactured McKibben actuators. Firstly, the electro-mechanical characteristics of the proposed sensor were obtained and a model to compensate its nonlinearities was evaluated. Secondly, an estimation of the accuracy was performed during dynamic actuator contractions. The results showed that, using this sensor, a direct measurement of the actuator axial displacement can be obtained within $20 \%$ error, without affecting its performances in terms of contraction.
\end{abstract}

\section{INTRODUCTION}

Fiber-reinforced fluidic actuators are very much used in the soft robotics field [1]. This class of actuators includes devices that can bend [2], twist [3], curl [4], and extend [5] upon pressurization. This group includes also devices that can contract along their length like biological muscles [6], known as pneumatic artificial muscles (PAMs) or McKibben muscles. The McKibben actuator is a pneumatically driven device consisting of a cylindrical flexible rubber bladder, sheathed with an inextensible fiber network. When pressurized the external braided sleeve due to its tensile strength, enables a muscle-inspired contraction [7]. This actuator is flexible, lightweight and has high force-weight ratio [8]. In addition, the flexibility of both the fibers and the bladder makes it fully compliant [9].

In recent years, the design and control of McKibben actuators have been prominent research areas, especially in the field of soft and wearable robotics. In fact, different works focused on healthcare applications such as rehabilitation, mobility enhancement, and prosthetics [10], [11], [12]. However, due to the complex material mechanics of McKibben actuators, they resulted to be more challenging to control than traditional actuator technologies. As such, soft inflatable actuators generally require external displacement sensors for feedback control. However, the commercialized

This work was partially supported by the European Commission through the Hybrid Heart Project under Grant 767195.

M. Maselli**, D. Zrinscak and M. Cianchetti are with the BioRobotics Institute, Scuola Superiore Sant'Anna, Pisa 56025, Italy (email: martina.maselli@santannapisa.it, debora.zrinscak@santannapisa.it, matteo.cianchetti@santannapisa.it).

V. Magliola is with the Department of Information Engineering, University of Pisa, Pisa 56122, Italy (email: vannimagliola@ gmail.com).

**Corresponding author (phone +39 050 883078; fax: +39 050883497 ; e-mail: martina.maselli@ santannapisa.it). conventional displacement sensors, i.e. potentiometer, linear encoders and so on, are relatively hard, bulky and heavy; therefore, attaching them to the pneumatic artificial muscles negatively affects their aforementioned advantages [13].

Consequently, there has been increasing interest in embedding sensing capabilities into McKibben actuators in order to produce more controllable soft devices. Wakimoto developed a McKibben actuator with a build-in flexible electro-conductive rubber sensor, which can measure the length by actuator itself [14]: the overall system contraction induces a measurable electrical resistance variation. However, for gaining the displacement from the flexible sensors, axial tension is need in default position. Another study has demonstrated the use of an embedded microchannel, filled with a conductive liquid (eutectic Gallium-Indium - eGaIn), in the elastomer layer of the actuator bladder [15]. Air pressure inside the bladder stretches the elastomer layer, reducing the cross-sectional area of the embedded microchannel while simultaneously increasing its length; this causes a resistance variation that can be related to the length of the actuator However, the microchannel requires a complex fabrication process that limits the selection of elastomers and fibers for the actuator. Goulbourne and Son, indeed, proposed to use dielectric elastomers to sense the PAM contraction [7]. Nevertheless, this approach limits the selection of the elastomer to thin materials with a high dielectric constant to achieve measurable capacitance. Another solution was to use insulated electrical wires as a replacement of the fiber network. The displacement can be calculated from the changes of the inductance of the conductive network. Although the fabrication process is relatively simple, the electrical wires must be similar to the actuator fibers in terms of dimensions and flexibility [1].

In the actuators described above, the actuation materials share elements with the sensing materials, which could limit the actuator device. Moreover, these embedded sensors result to be inapplicable to an existing actuator design. Another method is to add sensors externally, such as conductive rubber sensors around a McKibben actuator [16]. This flexible sensor was used to measure the radial expansion that has been related to the length variation geometrically. Such attachable sensors should be as thin as possible, so as not to disturb the deformation of a McKibben actuator.

The goal of this study is to develop a flexible and low-cost sensor that can be applied to an existing McKibben actuator, without affecting its range of deformation and motion. To this aim, sensing solutions based on smart textiles can provide a valid alternative because of their low cost, lightness, flexibility and capacity to adapt to different body structures [17]. Smart textiles are fabrics that can sense and react to mechanical, thermal, chemical, magnetic and electric stimuli, by means of extrinsic and intrinsic modifications [18]. Accordingly, this work proposes a flexible strain sensor based on conductive 
textile that can detect the deformation of McKibben actuators without affecting their advantages.

Firstly, we fabricated and tested the McKibben actuator to be sensorized; secondly, we characterized the sensor by extracting the useful electro-mechanical characteristics and by evaluating to what extent it affects the contracting abilities of the artificial muscle. Then, we evaluated a simple model to compensate its nonlinearities. Finally, we fixed the conductive textile sensor over the actuator and tested its performances as monitoring system, in dynamic conditions, i.e. actuator contracting at different speeds. The tests results underlined the advantages of this design, as the low-cost and the possibility to be implemented on any type of McKibben actuator without affecting the existing form factors or shapes.

\section{MATERIALS AND METHODS}

\section{A. McKibben fabrication}

The pneumatic artificial muscle, whose motion is analyzed in this paper, consists of an internal elastomeric chamber, obtained through silicone casting techniques, sheathed with an external PVC braided sleeve available commercially. To obtain the desired specifications of the chamber, e.g. compliance and small radial dimensions, a specific mold has been designed and 3D printed. The mold allows the simultaneous casting of ten silicone chambers: each one is characterized by an axial length of $55 \mathrm{~mm}$, an external diameter of $5 \mathrm{~mm}$ and a chamber wall thickness of $1 \mathrm{~mm}$, the employed material is Ecoflex ${ }^{\mathrm{TM}}$ 00-30 (SMOOTH-ON! Inc.). This elastomeric bladder was then placed inside a braided sleeve, characterized by PVC threads braided in a double helix. The bladder and the external sheath were linked one to the other through two casted rings of Smooth-Sil ${ }^{\circledR} 950$ (SMOOTH-ON! Inc): while the top end was used to connect the actuator to the air supply, securing the tubes with silicone glue (Sil-Poxy ${ }^{\circledR}$, SMOOTH-ON! Inc.), the bottom end was sealed with the same material employed for the rings. The final active length of the tested actuator is $45 \mathrm{~mm}$. Once pressurized, the combination of a low braiding angle, $25 \mathrm{deg}$ with respect to the longitudinal axis in our case, and an easily deformable elastomeric chamber, enabled good contraction properties, $20 \%$ on average, even at low supply pressures, i.e. 0.50 bar as a working pressure.

\section{B. Sensor characterization}

We developed a flexible displacement sensor made from piezoresistive textile. Among the available possibilities on the market, Electrolycra (Mindsets Ltd, United Kingdom) has been selected for this study. Previous works have adopted Electrolycra as a strain sensor in several applications, e.g. the sensorization of continuum soft robots to reconstruct their spatial configuration [19], or to reveal bending and force in a soft body [20], or as a wearable goniometer [21] [22].

Electrolycra looks and feels like ordinary lycra, but it is highly conductive since it is weaved with Silver plated $76 \%$ Nylon 24\% elastic fibers [19]. In particular, Electrolycra shows a typical knitted structure where each yarn strand is composed of multiple fibers running in parallel, and each fiber has a non-conductive core with a silver particle coating [21]. Its conductivity depends on how tightly it is stretched, thus if it is pulled its resistance increases. Electrolycra main characteristics, investigated in [21] and here used as starting point, include:

- better performances can be reached after pre-stretching of $200 \%$;

- it has a preferential direction along which resistance significantly changes and a major strain is possible, with a working range from $0 \%$ to $50 \%$ of strain.

The electro-mechanical tests were performed at room temperature by using a material testing machine (model 4464, Instron Inc., Norwood, MA), which allows to impose a desired strain $S$ to the textile. Each end of the sample was fixed mechanically using clamps with copper sheets and welded wires. These wires were connected to a data acquisition board (DAQ, National Instruments ${ }^{\circledR}$ ) to measure the electrical resistance values, which were simultaneously recorded for different strains with a sampling frequency of $10 \mathrm{~Hz}$. In particular, the resistance value $R$ was measured by means of a voltage divider, composed of the Electrolycra and a resistor with resistance $R_{l}=10.5 \Omega$ connected in series, with an applied voltage $V_{\text {in }}=3.2 \mathrm{~V}$.

Due to material preferential direction [19] [21], uniaxial cyclic strain tests have been conducted along this direction on a specimen $5 \mathrm{~mm}$ wide and with length $L_{0}=40 \mathrm{~mm}$ along the preferential direction. We carried out tests at different level of velocity in order to evaluate the influence of the strain rate on the sensor characteristics. These tests were performed by applying on the sensor five loading-unloading cycles (from 0 to $50 \%$ strain) at five different strain rates $\left(v_{l}=1 \mathrm{~mm} / \mathrm{min}, v_{2}\right.$ $=5 \mathrm{~mm} / \mathrm{min}, v_{3}=10 \mathrm{~mm} / \mathrm{min}, v_{4}=20 \mathrm{~mm} / \mathrm{min}$ and $v_{5}=$ $50 \mathrm{~mm} / \mathrm{min}$ ). The five cycles were averaged afterward.

Usually, piezoresistive strain sensors present remarkable electro-mechanical hysteresis. This is known as the major drawback of resistance-type sensors, which can limit severely their applicability. Several methods have been proposed to reduce the impacts of these factors [23] [24], but compensation through mathematical modelling is unavoidable to improve the stretch sensing accuracy [25] [26]. In this work tests at different strain rate were used to compensate for hysteresis, calculating two different fittings for loading $\left(R\left(t_{i}\right) \leq R\left(t_{i+1}\right)\right)$ and unloading $\left(R\left(t_{i}\right)>R\left(t_{i+1}\right)\right)$. This method introduces very small delays (just one sample) and allows to fit data from a wider range of speed solicitation with an improved accuracy [27]. The measurement accuracy with respect to the applied strain trends was analyzed in terms of root mean square error (RMSE).

\section{Sensor-actuator interaction characterization}

Static contraction experiments were conducted in order to understand if, and to what extent, the chosen sensing element characteristics were affecting the McKibben actuator contraction abilities. To this purpose, it became crucial to determine the length $L_{s}$ of the conductive textile stripes to be employed. Considering that the sensor can measure the amount of stretch, it had to be pre-extended, and, thus, the design constraints were the following: a) as mentioned in Section II.A, the difference between the natural length $L_{n}$ (null shrinkage) and the minimum one $L_{\min }$ (maximum shrinkage) 


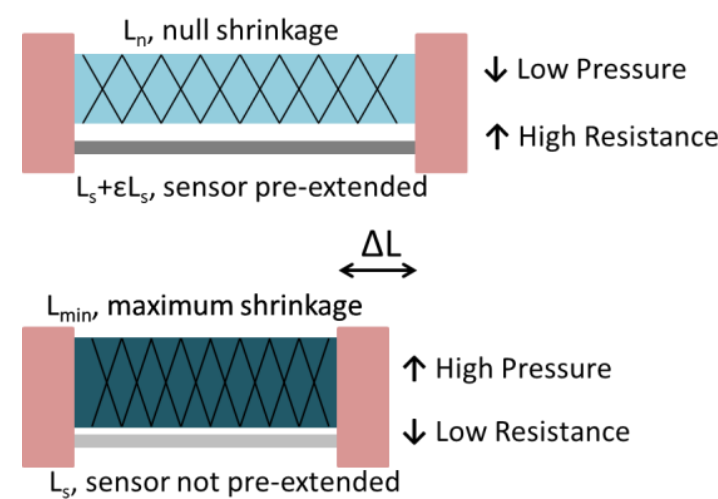

Figure 1. Schematic of McKibben actuator with the textile sensor in null shrinkage (top panel) and maximum shrinkage (bottom panel). $\Delta \mathrm{L}$ is the axial displacement.

of an actuated McKibben muscle (Fig. 1); b) as mentioned in Section II.B, the ability of the sensor to measure the amount of stretch within a specific extensional strain working range $\left(\varepsilon_{\max }=50 \%\right)$. In equations:

$$
\left\{\begin{array}{l}
L_{n}-L_{\min } \leq \varepsilon_{\max } \\
L_{n} \leq L_{s}+\varepsilon_{\max } L_{s}
\end{array}\right.
$$

Moreover, since the maximum shrinkage of the actuator is less than the maximum working range of the sensor, it is advisable to design the sensor size in order to use the first region of its resistance-strain characteristic, thus, exploiting a greater sensitivity and avoiding the plateau after which the resistance-strain behavior tends to decrease [21] [22].

Because of all the above considerations, the dimensions of the conductive textile stripes to be tested were chosen to be $40 \mathrm{~mm}$ in length and $5 \mathrm{~mm}$ in width (Fig. 1).

The actuator performances were evaluated under static conditions, by comparing the amount of contraction in the following configurations: a) without any sensors, b) with a single sensor fixed on a side, c) with two conductive textile stripes mounted symmetrically.

When needed, the sensors were attached using Parafilm "M"® (Bemis Flexible Packaging, Neenah, WI 54956) on the actuator ends. As it can be observed in Fig. 2, the sensor was attached to the sealing rings of the McKibben actuator without any rigid frame, as instead happens when mounting conventional displacement sensors.

In each configuration, the actuator was pressurized through an air compressor (Fiac air-compressors, Leonardo), coupled with an electronic valve (CAMOZZI, K8P-0-D522-08) to obtain steps of pressure values ranging from 0 to 0.55 bar. At each step, the driving pressure was checked through a digital manometer (CAMOZZI, SWCN-P10-P3-2).

The actuator shortening performances were video-recorded, and successively analyzed through the motion-tracking features of the Kinovea software: markers applied on the McKibben muscles ends helped the process.

\section{Sensor performance verification}

After selecting the single-sensor configuration, the accuracy and the performances of our soft sensor were

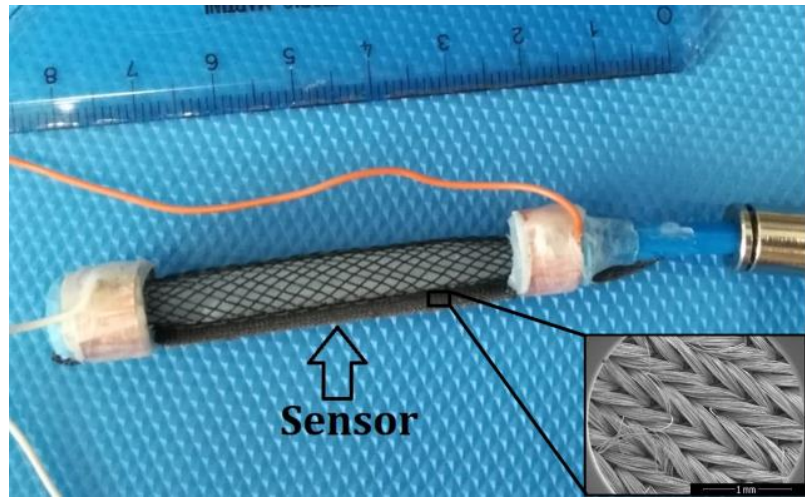

Figure 2. Textile sensor attached to a McKibben actuator (lower box: SEM image of the textile).

analyzed. The idea was to test it in terms of capability of monitoring random movements of the actuator, by varying its speed rate and axial deformation continuously. To generate the random motion, the actuator was supplied through the same setup described in Section II.C, exception made for the electronic valve that was removed from the circuit and substituted by a simple mechanical one.

To acquire the sensor voltage variation, wires were welded onto two copper tapes attached to the actuator ends. As shown in Fig. 2, the sensor was fixed on top of them by using Parafilm "M" (Bemis Flexible Packaging, Neenah, WI 54956). The wires were then connected to the same acquisition circuit adopted during the characterization phase.

After fixing two fiducial markers on the outer surface of the actuator, as in Section II.C, the tests were video-recorded. By processing each frame through the MATLAB ${ }^{\circledR}$ Image Processing Toolbox (MathWorks Inc., Natick, MA, USA), we were able to obtain an additional length measurement that was successively synchronized with the acquired ones through a MATLAB $^{\circledR}$ code. To evaluate the validity of the sensor measurements, the RMSE and Spearman correlation coefficient $\left(r_{s}\right)$ were evaluated on the actuator axial length calculated from both systems (sensor and video). The significance level was set at 0.05 .

\section{RESUlTS AND DisCUSSIONS}

\section{A. Sensor characterization}

The dependency on the strain rate is shown in Fig. 3. The relationship between strain and resistance obtained at $v_{1}, v_{2}, v_{3}$, $v_{4}$, and $v_{5}$ is hysteretic and, as the strain rate increases, the loop rotates counterclockwise around its low-left corner. Moreover, each loop is traveled clockwise, as marked by the arrows. This result is in agreement with the literature evidence [22] [28], and it is important for the design since it means that sensor could be used within a wide range of velocities, but with different calibration procedures.

This study aims at monitoring the axial length of the actuator during its dynamic contraction at a variable speed; therefore, it was necessary both a sensor calibration at a variable speed and one taking into account a range of strain rates. However, from Fig. 3 it is possible to notice that the variation between curves at different strain rate is visibly high and they cannot be related to the other ones without introducing considerable errors in the calibration and 


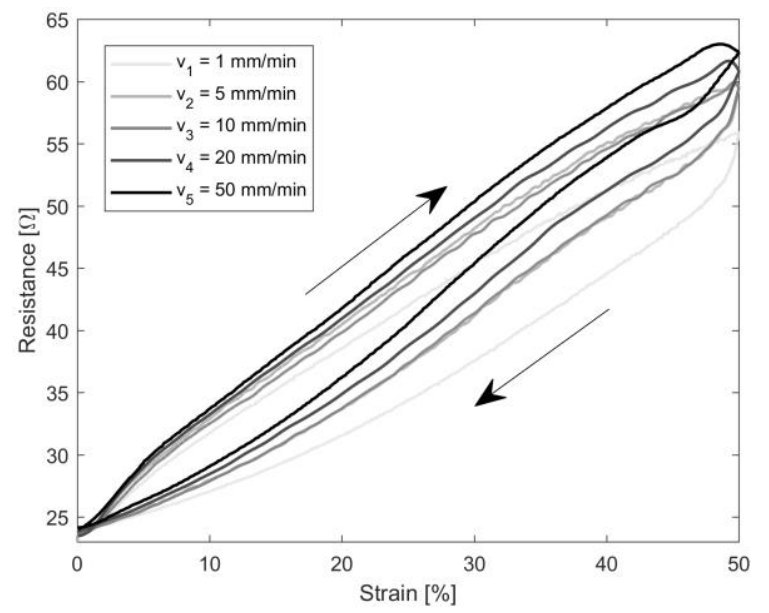

Figure 3. Sensor resistance at increasing strain rate.

hysteresis compensation of the sensor, as well as in estimating the axial length variation of the actuator. For these reasons, we used data from tests at $v_{1}, v_{2}$ and $v_{3}$ at the same time to compensate for hysteresis. Whereas, high strain rates $\left(\mathrm{v}_{4}\right.$ and $v_{5}$ ) should require further ad hoc calibrations that will not be evaluated in the present work.

As already mentioned, post-processing techniques were employed to compensate hysteresis. In the present work, two different calibration curves were considered, both resulting as polynomial fitting of the third order:

$$
S=c_{3} R^{3}+c_{2} R^{2}+c_{1} R
$$

where $R$ was expressed as a normalized relative resistance: $\left(R-R_{0}\right) / R_{0}$, with $R_{0}$ being the resistance at zero value strain. The first curve was calculated as the best fitting for the loading sections of the cycles at $v_{1}, v_{2}$ and $v_{3}$ and it had to be applied when the sensor was in a load phase (when $R\left(t_{i}\right) \leq R\left(t_{i+1}\right)$ ). The second curve was obtained as the best fitting for the unloading sections of the cycles at $v_{1}, v_{2}$ and $v_{3}$ and it had to be applied when the sensor was in an unload phase (when $R\left(t_{i}\right)>R\left(t_{i+1}\right)$ ).

A second model (used as benchmark) has been considered, which simply expresses the strain as a cubic function of the resistance (2), by neglecting the hysteresis. Coefficients $c_{1}, c_{2}$, $c_{3}$ were obtained through least squares optimization on the considered data and are summarized both for simple fitting and load/unload fitting in Table 1.

The calibration curves were applied on the tests at $v_{l}, v_{2}$ and $v_{3}$. Fig. 4 shows the RMSE values with the simple and load/unload fitting. As expected, the simple fitting leads to larger errors, since it does not represent hysteresis, whereas the load/unload fitting leads to minor errors. Results can be easily assessed from Fig. 5, confirming that an adequate postprocessing can enhance the sensor performances in case of dynamic measures. Fig. 5(a) shows the time evolution of the applied and estimated strains. It can be notice that the load/unload fitting is able to better estimate the strain at the three velocities. Fig. 5(b) shows the characteristics obtained with the simple (blue) and load/unload fitting (red) at the three velocities. The ideal characteristics of the sensor should present an estimated strain $(\hat{S})$ equal to the applied strain $(S)$.

If the simple fitting is used, hysteresis is not compensated, as demonstrated by the loops in the $(S, \hat{S})$ plane. With the load/unload fitting, the loop areas are greatly reduced,

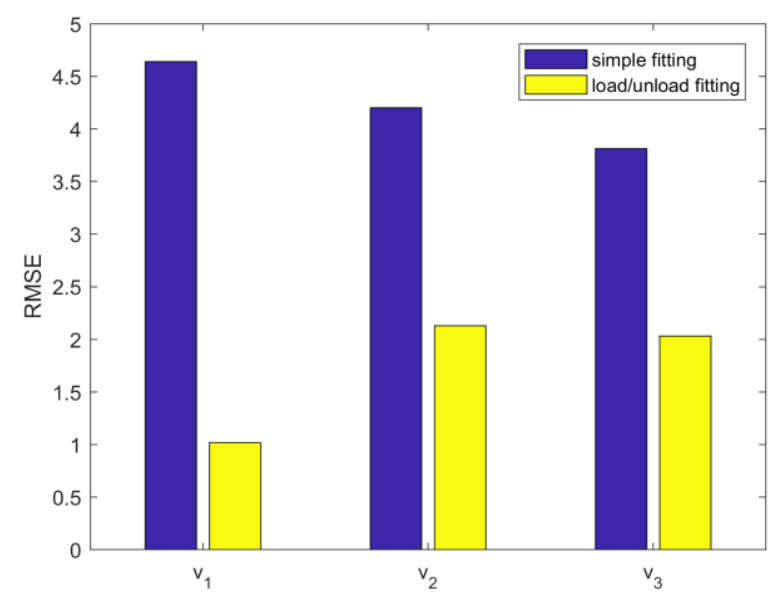

Figure 4. RMSE obtained on all the 3 velocities employing simple and load/unload fitting.

however the characteristics deviate from the ideal one (black dashed line) as the strain rate increases.

TABLE I. COEFFICIENTS PARAMETERS FOR SIMPLE AND LOAD/UNLOAD FITTING

\begin{tabular}{|l|l|l|l|c|}
\hline & \multicolumn{1}{|c|}{$\boldsymbol{c}_{\boldsymbol{1}}$} & \multicolumn{1}{c|}{$\boldsymbol{c}_{\boldsymbol{2}}$} & $\boldsymbol{c}_{\boldsymbol{3}}$ & $\boldsymbol{R}^{\mathbf{2}}$ \\
\hline Simple Fitting & 0.3118 & 0.0002699 & $-2.247 \mathrm{e}-06$ & 0.902 \\
\hline Load Fitting & 0.1507 & 0.001423 & $-3.146 \mathrm{e}-06$ & 0.9935 \\
\hline Unload Fitting & 0.385 & 0.0005442 & $-6.299 \mathrm{e}-06$ & 0.9889 \\
\hline
\end{tabular}

For these reasons, the above reported load/unload fitting at $v_{1}, v_{2}$ and $v_{3}$ has been used for the calibration of Electrolycra textile required to develop the sensor for monitoring axial displacement of McKibben actuator.

\section{B. Sensor-actuator interaction characterization}

After determining the most suitable length of conductive textile stripes, we performed experiments to evaluate in which measure their presence was affecting the motion of the fabricated actuator. As expected, at rest, in configuration (b) the presence of a single pre-stretched sensor introduces a slight bending towards the reinforced side. However, this effect, not visible in the other configurations, is completely canceled out after pressurization. Moreover, the actuator contractions, measured at each step through video analysis, revealed that configuration (b) and (c) introduce at maximum a relative error of respectively $1 \%$ and $3 \%$ with respect to the measurements acquired in configuration (a). These results confirmed the suitability of the proposed solution as sensing technology for a McKibben actuator, whenever lightness and compliance are a priority.

\section{Sensor performance verification}

In Fig. 6, the data acquired from our textile-based sensor and from video recording during tests are compared: the red line is the actuator estimation length calculated from our sensor, while the blue one is the true value measured by video recording. In the same figure, an overestimation of the higher length values and a more acceptable estimation of the lower length values can be noticed. This trend may be related to the sensor drift: in [21], results show that, when the textile is stretched, the initial overshoot and decrease (relaxation) of the 
resistance is particularly visible in response to a constant strain applied to the sensor.

Moreover, we observed that the developed sensor can better measure the axial length variations during the shrinkage of the actuator, which is equivalent to the sensor unloading phase. Whereas, low sensor performances were obtained in the loading phase of the sensor, i.e. when the actuator returns to its initial condition (null shrinkage). It is also important to notice that when the actuator decreased its velocity until it reached a steady state, the sensor measured the length of the actuator better when it was contracting. In particular, a more precise measurement was obtained when the actuator was in its maximum shrinkage, i.e. $39 \mathrm{~mm}$ (see Fig. 6).

The RMSE value was $1.27 \mathrm{~mm}$, about $20 \%$ of entire axial displacement of the actuator. This value is not negligible, but in line with other studies [16] [29]. The acceptability of this RMSE is related to the intended application. If the priority is to acquire more accurate actuator length measurements, a more complicated model should be applied to compensate for the hysteresis error and able to reproduce the relaxation dynamics (e.g. [30]). The disadvantage would be a higher computational cost to be used to acquire the deformation data.

In addition, the actuator length obtained from our sensor was positively correlated $\left(r_{s}>0.90\right.$ and $\left.p<0.05\right)$ with that measured by video recording. According to [31], Spearman's coefficient values were considered excellent if $r_{s}>0.90$, good for $r_{s}>0.75$, moderate for $r_{s}>0.50$. The correlation is thus statistically significant, which means that proposed solution could be considered valid for measuring the axial length of soft actuators.

The experiments, conducted in dynamic conditions, qualitatively proved the validity of the proposed solution as axial displacement sensor. Indeed, although the accuracy levels cannot be compared with the ones of the classic sensing

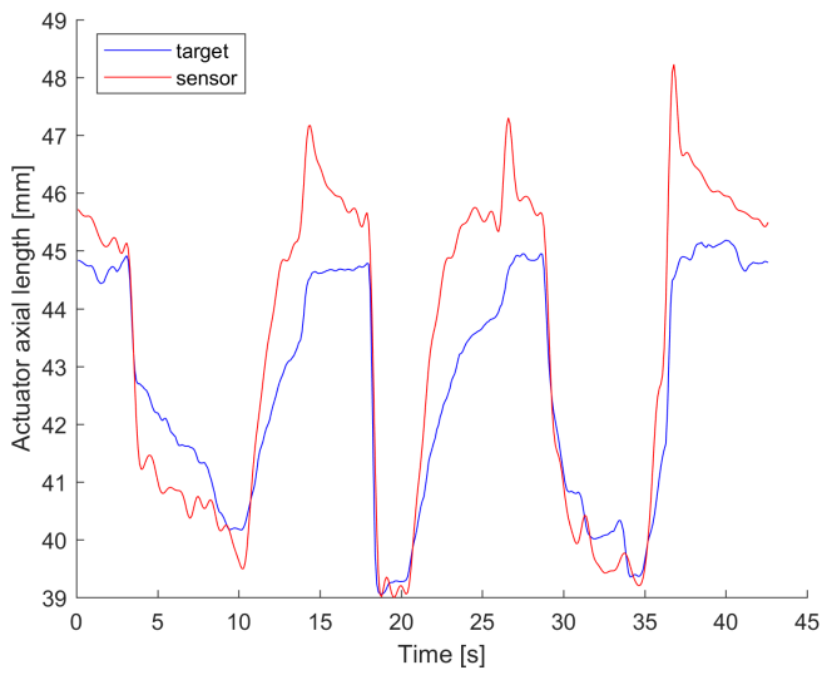

Figure 6. Estimated length from the flexible sensor (red) compared to true value measured from video recording (blue).

technologies, we were able to obtain satisfying results considering their level of innovativeness, compliance and lightness.

\section{CONCLUSIONS}

In this work, a flexible strain sensor based on piezoresistive fabric has been developed and integrated to estimate the axial displacement of McKibben pneumatic actuators. Because of its elasticity, highly conductivity, low cost, lightness and limited cumbersomeness Electrolycra was selected. In our study, we show that a simple hysteresis compensation model, characterized by a low computational cost, combined with an adequate processing, can enhance the sensor performances in case of dynamic measurements. Moreover, thanks to the
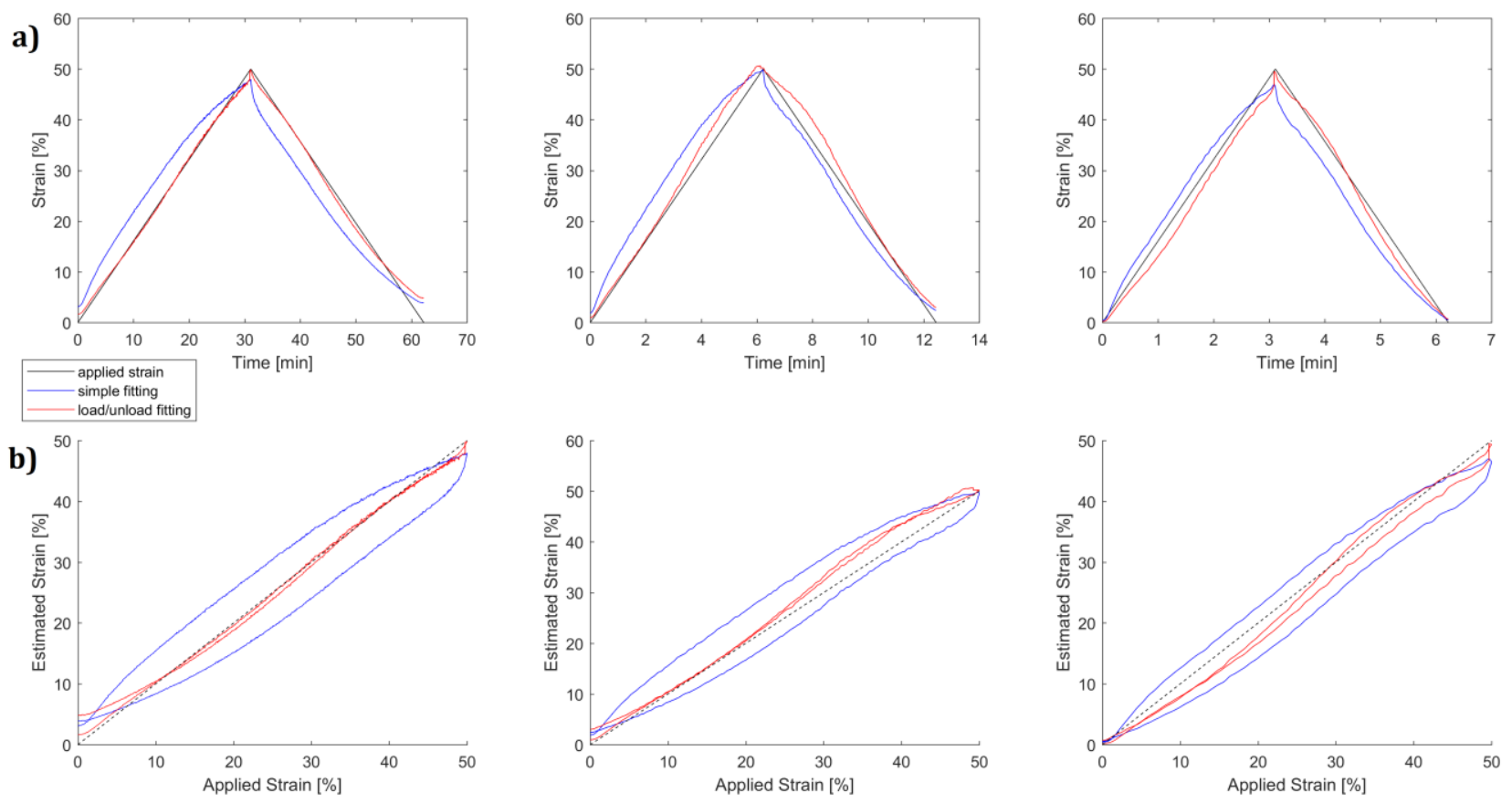

Figure 5. (a) Applied strain curves versus estimated strain curves calculated using both the simple (blue) and the load/unload fittings (red) for $v_{l}$ (left panel), $v_{2}$ (middle panel) and $v_{3}$ (right panel); (b) estimated strain $\hat{S}$ with simple and load/unload fitting as a function of the applied strain $S$. The black dashed line represents the ideal characteristic $\hat{S}=S$. 
electro-mechanical characteristics, we managed to obtain continuous measurements, almost without affecting the original range of deformation of the soft actuator. Although the sensed contraction accuracy level is still low, our solution allows to maintain the flexibility and compliance of the McKibben otherwise lost by employing traditional sensors.

In conclusion, this study laid also the foundations for, not only all the possible future improvements of the system, as the development of a more complex model to compensate the sensor nonlinearities, but also some important potential applications, e.g. its use as basic dynamic control strategy element suitable for a wide range of both traditional and soft actuators, where the reconstruction of all aspects of deformation is not necessary and features as compliance and lightness are the required.

\section{REFERENCES}

[1] W. Felt, K. Y. Chin, and C. D. Remy, "Contraction sensing with smart braid McKibben muscles," IEEE/ASME Transactions on Mechatronics, vol. 21, no. 3, pp. 1201-1209, 2016.

[2] K. C. Galloway, P. Polygerinos, C. J. Walsh, and R. J. Wood, "Mechanically programmable bend radius for fiber-reinforced soft actuators," in Proc. 16th Int. Conf. Adv. Robot., 2013, pp. 1-6.

[3] H. M. Paynter and J. Juarez, "Thermodynamic treatment of tug-\&-twist technology. 2. Thermodynamic twistor design," in Proc. IEEE/ASME Int. Conf. Adv. Intell. Mechatron., 1999, pp. 826-829.

[4] J. Bishop-Moser and S. Kota, "Towards snake-like soft robots: Design of fluidic fiber-reinforced elastomeric helical manipulators," in Proc. IEEE/RSJ Int. Conf. Intell. Robots Syst., 2013, pp. 5021-5026.

[5] M. D. Grissom, V. Chitrakaran, D. Dienno, M. Csencits, M. Pritts, B. Jones, W. McMahan, D. Dawson, C. Rahn, and I. Walker, "Design and experimental testing of the octarm soft robot manipulator," in Proc. Defense Security Symp., 2006, pp. 62301F-1-62301F-10.

[6] D. Caldwell, N. Tsagarakis, and G. Medrano-Cerda, "Bio-mimetic actuators: Polymeric pseudo muscular actuators and pneumatic muscle actuators for biological emulation," Mechatronics, vol. 10, no. 4, pp. 499-530, 2000.

[7] N. C. Goulbourne, and S. Son, "Numerical and experimental analysis of McKibben actuators and dielectric elastomer sensors," In ASME 2007 International Mechanical Engineering Congress and Exposition (pp. 175-185). American Society of Mechanical Engineers, January 2007.

[8] B. Kang, C. Kothera, B. Woods, and N. Wereley, "Dynamic modeling of mckibben pneumatic artificial muscles for antagonistic actuation," in 2009 IEEE International Conference on Robotics and Automation (ICRA2009), Kobe International Conference Center, Kobe, Japan, May 12-17 2009, pp. 182-187.

[9] O. Erin, N. Pol, L. Valle, and Y. L. Park, "Design of a bio-inspired pneumatic artificial muscle with self-contained sensing," In Engineering in Medicine and Biology Society (EMBC), 2016 IEEE 38th Annual International Conference of the (pp. 2115-2119). IEEE, 2016, August.

[10] Y.-L. Park, J. Santos, K. G. Galloway, E. C. Goldfield, and R. J. Wood, "A soft wearable robotic device for active knee motions using flat pneumatic artificial muscles," in Robotics and Automation (ICRA), 2014 IEEE International Conference on, 2014, pp. 4805-4810.

[11] D. Ming, J. Ueda, and T. Ogasawara, "Pinpointed muscle force control using a power-assisting device: System configuration and experiment," in Biomedical Robotics and Biomechatronics, 2008. BioRob 2008. 2nd IEEE RAS \& EMBS International Conference on, 2008, pp. 181-186.

[12] K. Kim, K.-J. Hong, N.-G. Kim, and T.-K. Kwon, "Assistance of the elbow flexion motion on the active elbow orthosis using muscular stiffness force feedback," Journal of Mechanical Science and Technology, vol. 25, pp. 3195-3203, 2011/12/01 2011.

[13] T. J. Yeh, M. J. Wu, T. J. Lu, F. K. Wu, and C. R. Huang, "Control of McKibben pneumatic muscles for a power-assist, lower-limb orthosis," Mechatronics, vol. 20, no. 6, pp. 686-697, 2010.
[14] S. Wakimoto, K. Suzumori and T. Kanda, "Development of Intelligent McKibben Actuator with Built-in Soft Conductive Rubber Sensor," 2005 IEEE/RSJ International Conference on Intelligent Robots and Systems, pp. 487-492, Aug 2005.

[15] Y.-L. Park and R. J. Wood, "Smart pneumatic artificial muscle actuator with embedded microfluidic sensing," Proc. IEEE Sens. Conf., pp. 689692, Baltimore, MD, 2013.

[16] S. Kuriyama, M. Ding, Y. Kurita, J. Ueda, and T. Ogasawara, "Flexible sensor for McKibben pneumatic artificial muscle actuator," International Journal of Automation Technology, vol. 3, no. 6, pp. 731740, 2009.

[17] F. Lorussi, N. Carbonaro, D. De Rossi, and A. Tognetti, Strain- and Angular-Sensing Fabrics for Human Motion Analysis in Daily Life. Cham: Springer International Publishing, 2017, pp. 49-70. [Online]. Available: https://doi.org/10.1007/978-3-319-50124-6_4.

[18] L. M. Castano and A. B. Flatau, "Smart fabric sensors and e-textile technologies: a review," Smart Materials and Structures, vol. 23, no. 5, p. 053001, 2014.

[19] M. Cianchetti, F. Renda, A. Licofonte, and C. Laschi, "Sensorization of continuum soft robots for reconstructing their spatial configuration," in Biomedical Robotics and Biomechatronics (BioRob), 2012 4th IEEE $R A S \&$ EMBS International Conference on. IEEE, 2012, pp. 634-639.

[20] C. Lucarotti, M. Totaro, A. Sadeghi, B. Mazzolai, and L. Beccai, "Revealing bending and force in a soft body through a plant root inspired approach," Scientific reports, vol. 5, p. 8788, 2015.

[21] A. Grassi, F. Cecchi, M. Maselli, M. R"oling, C. Laschi, and M. Cianchetti, "Warp-knitted textile as a strain sensor: Characterization procedure and application in a comfortable wearable goniometer," IEEE Sensors Journal, vol. 17, no. 18, pp. 5927-5936, Sept 2017.

[22] M. Maselli, E. Mussi, F. Cecchi, M. Manti, P. Tropea, and C. Laschi, "A wearable sensing device for monitoring single planes neck movements: assessment of its performance," IEEE Sensors Journal, vol. 18, no. 15, pp. 6327-6336, 2018.

[23] L. Wang, Y. Han, C. Wu, and Y. Huang, "A solution to reduce the time dependence of the output resistance of a viscoelastic and piezoresistive element," Smart Materials and Structures, vol. 22, no. 7, p. 075021, 2013. [Online]. Available: http://stacks.iop.org/0964$1726 / 22 / \mathrm{i}=7 / \mathrm{a}=075021$.

[24] O. Atalay and W. R. Kennon, "Knitted strain sensors: Impact of design parameters on sensing properties," Sensors, vol. 14, no. 3, pp. 47124730, 2014.

[25] M. R. Sunny and R. K. Kapania, "A hysteresis compensator based on a modified dynamic preisach model for conductive polymer nanocomposites," in IUTAM Symposium on Multi-Functional Material Structures and Systems, B. Dattaguru, S. Gopalakrishnan, and V. K. Aatre, Eds. Dordrecht: Springer Netherlands, 2010, pp. 87-94.

[26] K. Kuhnen, "Modeling, identification and compensation of complex hysteretic nonlinearities: A modified Prandtl-Ishlinskii approach," European journal of control, vol. 9, no. 4, pp. 407-418, 2003.

[27] I. Baldoli, M. Maselli, F. Cecchi, and C. Laschi, "Development and characterization of a multilayer matrix textile sensor for interface pressure measurements," Smart Materials and Structures, vol. 26, no. 10, pp. 104011, 2017.

[28] C. Mattmann, F. Clemens, and G. Tröster, "Sensor for measuring strain in textile," Sensors, vol. 8, no. 6, pp. 3719-3732, 2008.

[29] S. Hirai, "Measuring McKibben actuator shrinkage using fiber sensor," In Robot and Human Interactive Communication (RO-MAN), 2015 24th IEEE International Symposium on (pp. 628-633). IEEE, 2015, August.

[30] A. Oliveri, M. Maselli, M. Lodi, M. Storace, and M. Cianchetti, "Model-based compensation of rate-dependent hysteresis in a piezoresistive strain sensor," IEEE Transactions on Industrial Electronics, 2018. doi: 10.1109/TIE.2018.2884204.

[31] L. G. Portney and M. P. Watkins, Foundations of Clinical Research: Applications to Practice. Englewood Cliffs, NJ, USA: Prentice-Hall, 2000. 